\title{
Desde la narración: el diario reflexivo como herramienta de comprensión profesional
}

\author{
A narrative perspective: reflective journals as a tool for professional \\ understanding
}

\author{
María Inés Blanc \\ miblancfacultad@gmail.com \\ Universidad Nacional de Mar del Plata, Argentina \\ Silvina Pereyra \\ silvinapereyra79@gmail.com \\ Universidad Nacional de Mar del Plata, Argentina \\ Mercedes Brusadelli \\ mechisbrusadelli@gmail.com \\ Universidad Nacional de Mar del Plata, Argentina
}

Recepción: 29 Octubre 2019

Aprobación: 10Agosto 2020

Publicación: 01 Septiembre 2020

Cita sugerida: Blanc, M. I., Pereyra, S. y Brusadelli, M. (2020). Desde la narración: el diario reflexivo como herramienta de comprensión profesional. Archivos de Ciencias de la Educación, 14(17), e083. https://doi.org/10.24215/23468866e083
Resumen: Nuestro propósito es indagar a partir de biografías de graduados y graduadas, las representaciones que dejaron una traza en sus vidas, para comprender cómo estas se hacen evidentes en sus prácticas docentes. Nos focalizamos en analizar de qué manera las huellas de la formación se han puesto de manifiesto en el trabajo cotidiano a partir de haberlo puesto en palabras, y así los diarios reflexivos se constituyeron en nuestra fuente primaria. En este artículo nuestro objetivo radica en buscar las llaves que nos permitan descubrir el proceso de construcción subjetiva que una participante ha experimentado trabajando sobre su biografía escolar y de qué manera busca la transformación de su práctica docente en algo verdaderamente significativo. La metodología de investigación biográfico-narrativa nos permitirá comprender la construcción de representaciones y su búsqueda de sentido sobre lo vivido.

Palabras clave: Diarios de reflexión, Narrativa, Significatividad, Riografías escolares.

Abstract: Taking the school biographies of novel teachers as the object of our research, we purport to identify the representations that have left a trace in their lives and understand how these are materialised in their pedagogical decisions. It proves particularly interesting to focus on the way narrating life events raises awareness of the influence of these traces on the professional sphere, and to this purpose we have prioritised the use of reflective journals as the primary source of our study. In this article, our aim is to find the keys that allow us to uncover the subjective construction process that one of the participants has experienced while delving into her school biography and how she seeks to transform her teaching practice into something truly meaningful. The methodology of biographical-narrative research will allow us to interpret the construction of representations and the search for meaning in lived experiences.

Keywords: Reflective journals, Narrative, Meaningfulness, School biographies. 


\section{INTRODUCCIÓN}

El artículo se encuadra en los estudios del Grupo de Investigación en Idiomas, Educación y Formación Docente (GIIEFOD), que se desprende del Grupo GIEEC (Grupo de Investigación en Educación y Estudios Culturales) de la Facultad de Humanidades de la Universidad Nacional de Mar del Plata. Dentro de este marco institucional se viene desarrollando desde hace más de diez años un recorrido que ha tenido como eje de investigación la búsqueda de docentes memorables y las buenas prácticas docentes.

En los últimos años el foco del trabajo del grupo se puso en la construcción de categorías desprendidas de las narrativas del profesorado contando sus experiencias docentes, siendo la buena enseñanza un eje fundamental para toda la producción grupal. Cuando nos referimos a este eje, tomamos la perspectiva desarrollada por Fenstermacher y Richardson (2005), Litwin (1997) y Jackson (2002), quienes sentaron las bases de un tipo de enseñanza que promueve los procesos de construcción del conocimiento desde múltiples estrategias que privilegian la activa participación de las y los estudiantes en la negociación de significados, el desarrollo del pensamiento crítico, y la enseñanza para la reflexión; a la vez que busca generar ambientes de aprendizaje favorecedores a través del vínculo afectivo. De esta forma y siguiendo a estos referentes, es que se acuña el concepto de "huellas de la buena enseñanza", que representan a las profundas marcas que quedan registradas en la biografía escolar y se desprenden de las y los docentes mentores, perviviendo en quienes luego se convierten en docentes abriéndose camino también tanto en la vida como en el aula (Porta y Sarasa, 2014).

A partir de ese inicio, el grupo de investigación ha centrado sus trabajos en el análisis de relatos biográficos, profundizando en la búsqueda de aportes desde el enfoque biográfico-narrativo, dando lugar a la aparición de categorías nuevas que fueron complejizando y enriqueciendo los trabajos, como la idea de enseñanza y vida planteada como un trayecto/viaje odiseico, el humor como recurso didáctico, o la urdimbre gestada entre intelecto y afecto, entre otras.

Este recorrido del GIEEC ha fundado las bases para nuestro Grupo GIIEFOD, que nace con un trabajo referido a la búsqueda de las buenas prácticas a partir del análisis de biografías escolares de docentes noveles del Profesorado de Inglés de la UNMdP. Las técnicas que priorizamos fueron la entrevista en profundidad y el grupo focal con la meta de movilizar reflexiones sobre la buena enseñanza, identificar eventos significativos y deliberar sobre las propias prácticas docentes.

Los hallazgos obtenidos a través de estas técnicas generaron la necesidad de una indagación más profunda en algunas de las categorías que encontramos. Nuestra atención se focalizó en interpretar, siempre desde el análisis de las biografías escolares, aquellas percepciones y representaciones que tanta traza dejaron en las vidas de las participantes y que hoy se hacen evidentes en sus prácticas; para lo cual implementamos el diario de reflexión.

En este artículo nuestros objetivos radican en buscar las llaves que nos permitan descubrir algunas de las trasformaciones que una de las participantes, Lourdes, ha experimentado trabajando sobre las huellas de su biografía escolar. También cuáles han sido sus esfuerzos intencionales para modificar la manera de trabajar con sus estudiantes, y de qué manera busca la transformación de su práctica docente en algo verdaderamente significativo.

\section{Perspectiva teórica}

A lo largo de nuestra trayectoria en el GIEEC, y ahora GIIEFOD, hemos recurrido a la metodología biográfico-narrativa, y en este estudio en particular recurrimos al uso instrumental de la narrativa, es decir, como insumo de reflexión.

El pensamiento narrativo es inherente al ser humano, al contar historias sobre sí mismo el sujeto se involucra desde lo emocional y lo intelectual en la búsqueda de sentido; poner en palabras lo vivido corporeiza la experiencia y es posible constituirla como objeto de análisis. A través de la narrativa construimos nuestra 
subjetividad, contamos fragmentos significativos de nuestras vidas y "damos unidad a nuestra existencia entendiéndola como la expresión de una historia singular que se despliega y desarrolla” (Polkinghorne, 1988, p.150). Narramos sobre nosotros para nosotros mismos, de manera que esa historia queda encerrada en otra, y cuando lo hacemos para los demás, esa historia queda a su vez encerrada en otra (Bruner, 1991). Para comprender esta idea de historias dentro de otras historias, nos remitimos al carácter recursivo de la narrativa. Cada vez que una historia es contada y recontada, adquiere vida propia: el yo se percibe como una audiencia cambiante creada por los relatos y para quién se cuentan esos relatos del yo; pero también la interacción con diferentes interlocutores/as dará lugar a nuevas historias que se irán co-construyendo con las contribuciones de los y las participantes (Van Langenhove y Harré, 2016). Entonces, el yo narrador, el yo protagonista y el yo audiencia (Bruner, 1991) quedan plegados en esas tramas, y al ponerlas en diálogo inter e intraindividualmente, se van develando los significados profundos y vamos forjando nuestra identidad, nuestra visión del mundo y el lugar que ocupamos en él.

Así, el relato encierra múltiples y complejas interconexiones entre la evocación, la emoción, el pensamiento, el lenguaje, y la enunciación (Souto, 2016). Cuando contamos historias de vida, lo hacemos con un esfuerzo intencional, elegimos qué y cómo contar, y ese discurso ofrece distintos niveles de lectura. Bruner (1991) parte de la premisa que "para comprender al hombre, es preciso comprender cómo sus experiencias y actos están moldeados por sus actos intencionales" (p.47), pero siempre teniendo en cuenta que dichos actos responden a los cánones de un sistema cultural en el cual nos hallamos inmersos. El autor se refiere a tres dimensiones claves para la interpretación de la narración: la esquematización o elaboración de marcos, la regulación afectiva, y el uso del lenguaje.

Para la primera dimensión, elegimos referirnos a van Dijk (1997a), quien ha desarrollado la teoría del contexto para el análisis del discurso. En el caso que nos ocupa, el discurso narrativo se encuadra dentro de un contexto social que le da sentido y conforma el marco contextual. El individuo pertenece a un grupo social ordenado y organizado esquemáticamente cuya existencia se basa en la conservación de normas, valores, creencias, intereses, y actitudes que lo conforman; todos estos marcos estructurantes de algún modo dominan la visión del mundo del grupo y, por lo tanto, sus formas discursivas. Consecuentemente, para comprender el discurso no sólo se deben analizar las categorías semánticas, sino las condiciones de producción del texto en su contexto social, sus funciones, propósitos comunicativos, el tipo de soporte, y el rol asignado a sus participantes, y "así asociar el discurso y el orador con la estructura social y la cultura" (van Dijk, 1997b, p.39).

Podríamos aseverar que las siguientes dos dimensiones se desprenden de la primera. La regulación afectiva, según Bartlett (1932; en Bruner, 1991), influye directamente en nuestra memoria ya que cuando intentamos recordar se movilizan emociones que en cierta medida determinan qué fragmentos de nuestra historia elegimos contar y cómo. El modo de narrar es una función retórica que nos posiciona como protagonistas y modela nuestras intenciones al reconstruir el pasado. En esta misma línea, el uso del lenguaje asume un rol preponderante: ¿qué tono elijo darle al relato? ¿Qué mirada quiero suscitar? ¿A quién le cuento mi historia? ¿Qué función retórica deseo cumplir (emotiva, informativa, estética)? Las respuestas a estas preguntas definen el uso del lenguaje en cuanto a registro (formal, informal), léxico, figuras retóricas (por ejemplo, metáforas), interjecciones, oraciones enunciativas, entre otros.

Consecuentemente, interpretar las reflexiones sobre lo acontecido que las personas eligen contar como parte relevante de su historia implica explorar el discurso desde distintas perspectivas. Souto (2016) compara la narración con una trama cuya textura de líneas, nudos y huecos guían la indagación: las líneas ofrecen secuenciación, se va tirando de los hilos para seguir la búsqueda de sentidos; los nudos nos señalan contradicciones, zonas problemáticas que invitan a la profundización; y los huecos nos atraen a develar las relaciones entre lo dicho y lo silenciado.

En el camino que hemos optado trazar desde la narrativa a la reflexión, definir el concepto de reflexión es quizás la tarea más ardua dada la variedad de modelos para abordarla en la formación docente. Como marco referencial para este estudio recurrimos a Bengtsson (1995), quien establece una interacción entre 
dos amplias categorías: autorreflexión y pensamiento. La primera se refiere a una búsqueda introspectiva de reflejarse, volver sobre sí mismo y descubrirse como persona; la segunda supone una actividad intelectual que implica meditación, examinación, escudriñamiento, análisis. Al pensar en nuestra propia práctica profesional, la autorreflexión pareciera ser una condición sine equa non para el pensamiento, su potencial epistemológico radica en que facilita los procesos de comprensión de la práctica y permite a las y los docentes tomar posición ante las situaciones problemáticas, todo esto coadyuvado por la función de distanciamiento que conlleva la autorreflexión (Bengtsson, 1995).

Desde este punto de vista, la reflexión en el campo de la educación es percibida como un proceso de conocimiento de un símismo como profesional. Cuando los y las docentes piensan en su actividad profesional, lo hacen desde lo autorreferencial, pero este mero acto no necesariamente lleva al conocimiento. Para ello, el sujeto debe distanciarse del fenómeno, indagarlo, y tomar conciencia de sí mismo revelándose como participante activo de manera de poder llegar a conclusiones. En definitiva, un proceso en el cual el sujeto se implica en replegarse, mirarse y pensarse como profesional adoptando una actitud crítica para analizar su práctica docente y la interrelación de esta con el campo disciplinar.

Para delimitar nuestro abordaje de la reflexión, nos apoyamos en el esquema de Calderhead (1989) que contempla los siguientes aspectos: la naturaleza del proceso, el contenido, las condiciones en las que se reflexiona y el producto. En este artículo, pensamos la reflexión como proceso de pensamiento para traer a la conciencia los aprendizajes implícitos, con el objetivo de identificar las huellas de la biografía escolar en la práctica actual, para lo cual priorizamos los diarios de reflexión como dispositivo. La función que le adjudicamos al proceso es elucidar las motivaciones y las representaciones preexistentes que se ponen en juego al enseñar. El producto al que aspiramos es la transformación del sujeto en los términos que explicamos a continuación.

Souto (2016) identifica cuatro rasgos distintivos de la reflexión que nos parecen pertinentes para analizar los diarios de reflexión. En primer lugar, su recursividad hace referencia a la naturaleza espiralada del proceso, donde se vuelve sobre la experiencia y cada vez que se la revisita su recuerdo es tamizado a la luz del presente haciendo lugar a nuevas lecturas para su análisis. Así, lo vivido se va enriqueciendo y cobra nuevos significados a medida que se revive y se repiensa. Cuando hablamos de transformación, entonces, nos referimos a ese sujeto que es atravesado por la experiencia y emerge de ella renovado.

El segundo rasgo es la temporalidad. La reflexión actúa como un hilo conductor enlazando el pasado vivido, el significado que se le atribuye en el presente, y su proyección a futuro que conlleva una transformación. Por consiguiente, no es un acto espontáneo e inmediato, requiere tiempo y sistematización para echar luz sobre los eventos. Siguiendo a Dewey:

Donde hay reflexión hay suspensión. El objeto de pensar es ayudar a alcanzar una conclusión, proyectar una terminación posible sobre la base de lo que ya está dado (...) El pensar es un proceso de indagación, de observar las cosas, de investigación. Es buscar, averiguar algo que no se tiene a mano (1998, p.131).

Dada la complejidad del proceso, surge la necesidad de la articulación de enfoques para abordar las múltiples aristas de los objetos de reflexión desde lo individual, lo interpersonal, lo institucional, lo psíquico, lo social, lo político. Souto (2016) plantea así que dicha articulación rehúye la simplificación del análisis a la vez que visibiliza las diferentes capas que subyacen en un mismo material.

Ninguno de los rasgos anteriores es posible si no existe la implicación de la persona en el proceso. Un sujeto comprometido con su formación que busca respuestas, que indaga, y que al pensar en lo vivido se reconoce partícipe responsable y se replantea nuevas metas. En fin, emulando el juego de palabras que hace Souto (2016) a partir del término 'pliegues', un individuo capaz de replegarse, pero también de desplegarse y transformarse.

En referencia al concepto de transformación, explicitaremos brevemente cómo lo concebimos desde una perspectiva pedagógico-didáctica. En la formación psíquica, desde el psicoanálisis, el sujeto se mueve entre un 
sistema consciente y otro inconsciente, con procesos y energías que son propios y muchas veces en conflictos entre sí. En la formación hay mecanismos de la consciencia ligados a la palabra y la conformación del yo, hay recuerdos que pueden ser evocados porque están implícitamente presentes durante la actividad mental. Entonces vemos que hay un trabajo en la interioridad psíquica del docente que puede ser de rechazo y resistencia, no tratados en la formación y que pueden ser la manifestación del malestar o bienestar en la profesión incluso en las relaciones de saber que transfiere a sus alumnos. Si lo pensamos etimológicamente, transformar es cambiar la forma, mutar. La formación es un proceso dinámico, y la reflexión es la que nos permite focalizar en los procesos en que se producen modificaciones. Es a través de su escritura que la participante nota su transformación a la vez que en el proceso de leerla nosotras también lo percibimos y damos cuenta de ello (Souto, 2016).

\section{Diseño METODOLóGico}

El objetivo que perseguimos en este estudio es interpretar algunas de las transformaciones que una docente novel ha experimentado en su práctica actual a partir de haber identificado y reflexionado sobre las trazas que la han marcado en su biografía escolar.

Originalmente en nuestro primer Proyecto, trabajamos con un grupo de docentes noveles graduadas del Profesorado de Inglés de la Universidad Nacional de Mar del Plata, quienes habiendo cursado la Residencia Docente y Metodología de la Investigación -asignaturas en las que algunas integrantes del grupo de investigación nos desempeñamos- se sumaron al proyecto en una búsqueda de ahondar en su interés por la formación docente y la investigación educativa. Buscamos perfiles diferentes según franja etárea, historia de vida, contexto profesional, porque justamente intentábamos encontrar huellas en las biografías que nos permitieran profundizar en nuestros hallazgos a través de las narrativas.

El primer criterio de selección que consideramos fue una antigüedad docente no mayor a tres años porque de acuerdo a las investigaciones sobre el tema, son las y los docentes noveles quienes suelen apelar a los modelos aprehendidos experiencialmente a través de sus historias escolares al enfrentarse a nuevas problemáticas a resolver en el aula. Otro criterio vital para el proyecto fue la implicación de las participantes en el proceso; su compromiso y disponibilidad para la participación en las actividades planteadas durante las diferentes etapas de la indagación. Finalmente, cuatro docentes manifestaron su intención de continuar en el proyecto, y este artículo se centra en una de las participantes, a quien identificamos con un seudónimo: Lourdes.

La investigación biográfico-narrativa parte de una mirada cualitativa que no exige los parámetros cuantitativos para la selección de los participantes: la intuición, la emoción, los sentimientos, forman parte de nuestro campo de trabajo y por lo tanto también de la selección del grupo con el que investigamos. La muestra es pequeña, intencional y relevante a los objetivos del estudio.

En el trabajo utilizamos metodología de investigación cualitativa, y dentro de esta perspectiva, la hermenéutica y el método biográfico-narrativo. Es que nuestro interés radica en la manera de interpelar a la información recibida, lo que nos permite dar cuenta del proceso complejo en el entramado de relaciones que se producen al interior de esa construcción individual cargada de subjetividad.

Erickson (1989) nos remarca que la investigación cualitativa que centra su atención en la enseñanza es una de las más recientes dentro de este campo de investigación, para la cual las preguntas claves son: “¿Qué está sucediendo aquí, específicamente? ¿Qué significan estos acontecimientos para las personas que participan en ellos?” (p. 6). Es decir, trabajamos desde un proceso de comprensión de las representaciones que las personas involucradas tienen y vamos desde lo particular a lo general buscando detalles que nos permitan encontrar su correlato teórico.

Con respecto a la perspectiva narrativa, adoptamos las ideas planteadas por Bruner (1991), Hyvärinen (2010), y Bolívar, Domingo y Fernández (2001), considerando que las vidas de estudiantes y de docentes que hablan de sus experiencias constituyen material legítimo como fuente de elaboración de nuevos saberes 
acerca de lo educativo y lo social. La investigación narrativa utiliza como instrumento principal la entrevista, que fue nuestro insumo en el primer proyecto. Allí vimos cómo las docentes involucradas nos revelaban el entramado íntimo personal y profesional contando su vida. Luego realizamos entrevistas de grupo focal y por último en este segundo proyecto nos centramos en los diarios de reflexión.

Estos diarios constituyen una fuente de información dentro de la indagación narrativa que pueden ser escritos para diferentes propósitos. Como herramienta de análisis son muy completas porque permiten a quien las escribe reflexionar y darse cuenta de los procesos internos que han impactado en sus vidas y en su profesión. El propósito de solicitar a las participantes la elaboración del diario de reflexión fue justamente ver de qué manera el producto de las entrevistas y el grupo focal que habíamos realizado se había puesto de manifiesto en el trabajo cotidiano con un formato más explícito a partir haberlo puesto en palabras. Para ello, diseñamos un guion (ver anexo) con el propósito de facilitar el proceso de reflexión y orientar la búsqueda de significados. En ese guion citamos a Marta Souto (2016), quien en su libro Pliegues de la Formación. Sentidos y herramientas para la formación docente inicia su relato autobiográfico de manera narrativa con el claro objetivo de pensarse como sujeto en formación y a la vez como formadora. Desde ahí es que busca en sus propias huellas aquello que la marcó en estos dos sentidos como sujeto. En sus palabras:

[...] bucear en el pasado las motivaciones, las marcas, las huellas que otros trazaron y que se inscribieron en mí, dejando el interés profundo por la formación, su estudio y sus prácticas; romper el silencio de la página en blanco para, contando mi historia, invitar a los lectores a que escriban la suya. A que indaguen en ella... (p.25).

Esta escritura reflexiva es la que vemos a partir de la autora y de las palabras de Lourdes como un derrotero de construcción del yo persona y yo docente, reflexionando sobre el pasado e iluminando el porvenir. Dice Souto "... escribir es abrir un camino en el recordar, revivir, y a la vez volver, para pensar y construir desde otro lado...” (2016, p.25).

En el mismo sentido, Alsina y Batllori (2015) sostienen que el proceso ideal de reflexión docente se basa en "una alternancia entre acción y reflexión" (p.7). Los autores explican la importancia de ser capaces de observar y narrar nuestras experiencias previas consciente y reflexivamente.

Estas son las herramientas metodológicas con las que analizamos el diario de Lourdes y al hacerlo nos permitimos volver a narrar desde nuestras propias concepciones para intentar entender. Vemos cómo Lourdes se sigue transformando mientras piensa y redacta, mientras evoca y ejecuta una propuesta de clase. Y a nuestros ojos se la ve crecer como docente, pero porque también crece como persona.

\section{Nuestro CAMino de Deconstrucción}

Como parte del trabajo con nuestras entrevistadas, hemos pedido a las cuatro docentes participantes en nuestra investigación que escriban un diario reflexivo guiado por ciertas consignas de escritura plasmadas en un guion que adjuntamos en el anexo. Hemos seleccionado el trabajo de Lourdes, ya que nos ofrece un texto potente que nos moviliza a la escritura de este artículo. A continuación, transcribimos la narración que hemos realizado a partir de la lectura de su diario reflexivo.

\section{Lourdes: primer momento}

La primera parte del diario de reflexión solicitó a las participantes una apreciación personal en torno a cómo contribuyó su participación en el Proyecto Huellas a su práctica profesional. La implicación de Lourdes en el proceso de reflexión para volver a pensarse y narrar sobre sí misma, invita a pensar en ella, en su formación y sentimientos, y en luego volver a narrarla.

Hay muchas cosas que surgen de la lectura de Lourdes, algo que capta nuestra atención es que siente que todo este proceso de participación como sujeto de investigación ha sido una experiencia enriquecedora que 
le ha posibilitado repensarse, ya que manifiesta que "el valor de la propia historia de vida, contada, relatada, escrita, narrada nos transforma sobre todo como docentes". Así, contar, relatar, escribir, la transforma. La narrativa de este breve diario invita a nuestra participante a mirarse introspectivamente, pero como investigadoras y formadoras naturalmente nos vemos reflejadas en sus pensamientos, existe una conexión inmediata con las propias experiencias.

De su texto se desprende el pensarse diacrónicamente a sí misma y también a los demás, menciona al “otro histórico" y al "otro de hoy”: ¿Cuánto había? ¿Cuánto cambia? ¿Cuánto queda? ¿Realmente somos capaces de pensarnos? ¿O hablamos desde lo que nos gustaría? Una idea interesante es el pensarse como sujeto social en el proceso de construcción de significados con los otros.A quién marco y quién me marca,es reconocerse como portador de experiencias que han dejado su traza, pero también como persona proactiva capaz de transformar la realidad y dejar huellas. Al expresar que se siente atravesada en su rol docente, se advierte el compromiso en la labor, los acontecimientos no pasan casualmente, sino que "nos" pasan y nos movilizan. Por ello, repensarse conlleva darse cuenta de las decisiones que se han tomado, como escribe Lourdes, "descubrir aciertos y errores" e identificar "hasta profundas dudas", lo que desencadena el potencial heurístico de la reflexión.

Lourdes hace explícito cómo en el acto de narrar el yo narrador, el yo audiencia y el yo protagonista quedan plegados en las historias: "no sólo me repienso en relación a mi biografía, sino también en cómo la narré a otros". Esto nos recuerda lo difícil que es ponerse en un lugar de observador de uno mismo, objetivarse, objetivar recuerdos, relaciones, situaciones... ¿Quiénes somos? ¿Cómo llegamos hasta aquí? ¿Es casual nuestra intervención? ¿Cuánto de lo que se nos pide escribir es real objetivamente? ¿Cuánto está tamizado por el recuerdo?

El carácter de recursividad de la narrativa queda evidenciado cuando nuestra participante expresa: "no solo tomo conciencia de cómo mi historia contribuye en mi práctica sino cómo los relatos que doy de ella lo hacen”, y entonces así se pone de manifiesto la subjetividad de la narración y la potencia que le damos a lo que creemos importante para la situación comunicativa específica.

Mi narrativa "contribuye a veces muy inconscientemente, pero la veo en el modo de relacionarme con mis estudiantes y la dinámica de mis clases". Es decir, pensarnos y escribir sobre ese pensamiento inevitablemente deja marca.

\section{Lourdes: segundo momento}

Para reflexionar acerca de su práctica profesional, Lourdes selecciona un grupo porque siente que son más receptivos y amenos. Sin embargo, a medida que escribe va tomando distancia y discierne nuevos significados que quizás subyacían a nivel inconsciente.

El curso seleccionado es un $6^{\circ}$ año de Educación Primaria en una escuela privada: es el mismo grupo de niños que conoció en su primera experiencia cuatro años atrás en $2^{\circ}$ año. $\mathrm{Al}$ respecto, asegura: "me resultó bastante motivador volver a encontrarme con estos estudiantes dado que ellos me habían acompañado cuando estaba mucho más llena de incertidumbres que de certezas que hoy en día". Al tiempo que resalta que la Formación del Profesorado de Inglés no prepara a los futuros docentes para trabajar con niños pequeños de Primer Ciclo, sus palabras reflejan cómo el desafío de tal experiencia la ha transformado.

Inicialmente, asegura sentirse motivada a trabajar con este grupo porque los conoce. Escribe: "me gusta la idea de profundizar en la motivación para enseñar y no sólo como herramienta para dar clase”. ¿Qué es lo no dicho en esta frase? ¿Qué es lo no dicho en la selección del curso? Nos animamos a pensar que de las relaciones más amables en términos humanos se obtienen mejores resultados académicos. La relación estudiantes-docente y docente-estudiantes, es decir en ambos sentidos, es absolutamente equilibrada, no hay relación de poder que pueda marcarla afectivamente. Que las relaciones de poder existen es cierto, pero los 
resultados son diferentes cuando son desiguales. También en la frase hay una gran carga de interés por el otro en tanto persona, quiere motivar a sus alumnos en el sentido ético de que algo sea válido de ser aprehendido.

A medida que explica su clase, del desarrollo se desprende que planifica de la misma manera para cursos paralelos pero que va adecuando los contenidos de acuerdo con la respuesta del grupo y debe generar otras dinámicas porque su planificación toma menos tiempo para estos niños a quienes denomina "fast finishers". No los mira sólo como estudiantes, ve en ellos las cualidades que evidentemente la atraen humanamente, el respeto por la heterogeneidad, la solidaridad del grupo y su entusiasmo.

De manera recurrente insiste que estos niños la motivan a enseñar. ¿Cuánto de ello tiene que ver con el grupo en sí, como grupo escolar, o con los lazos que se han creado entre todos ellos incluyendo a Lourdes? ¿Podemos separar una cuestión de otra? Lourdes habla de espacios compartidos, de contar situaciones cotidianas en sus vidas, lo que ha derivado en un momento de la clase transformado didácticamente en un "storytelling". ${ }^{2}$ A pesar de que a veces piensa que cuenta demasiado de su vida, el gran vínculo afectivo hace que, en sus propias palabras: "nos contamos cosas, nos dejamos entrar en nuestras vidas".

Tal como le solicitamos, diseña una actividad para esta etapa de registro y organiza un proyecto de juego de roles en un restaurant con situación problemática. Definitivamente es muy interesante pensar en la problematización, salir de los estereotipos de enseñanza de la lengua y dar un giro complejizador en el sentido pedagógico. Lourdes se muestra orgullosa de sus estudiantes, quienes trabajan colaborativamente respetando las potencialidades de cada uno y generando propuestas muy creativas. Es en ocasión de describir estas clases cuando aflora el verdadero motivo para la selección del grupo: "quizás lo elijo porque no es nada como lo que yo viví en la primaria”.

En este sentido, al bucear en la relación entre su biografía escolar y su práctica profesional actual, no tiene mucho para agregar diferente a lo trabajado a través de su entrevista y del grupo focal. Ella continúa manifestando su experiencia negativa en la escuela primaria, y adjudica su memoria esquiva a la interrupción de la escolaridad por constantes viajes al hospital en la ciudad capital. No hay puntas de dónde tirar para desatar esos nudos en la trama narrativa. Lourdes se ve de alguna forma forzada a pensar en cómo su práctica se distancia de esos momentos que recuerda con tristeza y en intentar que esas experiencias negativas se transformen, empatizando -según sus palabras- con las "Lourdes" que encuentra en su trayecto como docente: chicos accidentados o con problemas de movilidad que ahora son sus estudiantes. Y finalmente no responde a la consigna planteada para el registro del diario de reflexión. ¿No hay más para decir? Intuimos que es doloroso volver a pensar sobre el tema.

Cuando preguntamos sobre las similitudes o diferencias entre la clase seleccionada y su propia biografía escolar, Lourdes retoma algo ya dicho: su práctica se enriquece cada vez que se plantea pensarse a sí misma. Ese mecanismo le genera la necesidad de ser activa y contenedora. Sobre este aspecto agrega algo antes no mencionado: al contrario de su trayecto escolar, su recorrido universitario y de militancia estudiantil han sido contenedores. Lourdes se posiciona en el relato con un sentido de agencia en su propia historia. Intelectualmente inquieta, nos cuenta sobre su compromiso político, sus estudios de posgrado, y se ha apropiado del léxico pertinente al campo de la investigación biográfico-narrativa. Así, su diario pone en diálogo la automotivación profesional, la reflexión, y la metacognición.

Hacia el final reflexiona sobre la necesidad de que sus estudiantes la vean con capacidad de entenderlos y acompañarlos, como sujetos activos y que se transforman. Sin dudas, el diario de Lourdes nos invita a pensarla en un marco de referencia más amplio donde la formación no lo es todo, la historia de vida, las marcas dolorosas o no modelan nuestra capacidad de trabajo educativo. El desafío es transformar esas marcas en un valor positivo hacia una profesión desafiante como la docencia. 


\section{Para Seguir Pensando}

En la forma de compartir el diario de Lourdes había caminos diferentes y nosotros tomamos una decisión que fue no seleccionar partes de su relato para hacerlos dialogar con los autores. Elegimos plantear los hallazgos de manera narrativa, deconstruyendo, haciendo también nosotras una interpretación de sus palabras, y buscando significados o haciéndonos cuestionamientos que nos permitieran comprenderla; el camino de la doble hermenéutica. Giddens, uno de los pioneros en el desarrollo de la metodología interpretativa en las ciencias sociales, valora "la historia no como transcurso del tiempo sino como su capacidad de llegar a ser conscientes de su propio pasado y de incorporar esa consciencia como parte de lo que es la historia" (Giddens, 1982, p.6).

Nosotras fuimos testigos de la mirada introspectiva de Lourdes, y cómo fue haciendo su propia interpretación de las razones de sus decisiones. Y mientras éramos testigos a partir de la lectura, nos animamos a hacer una nueva interpretación desde nuestra propia subjetividad al contarla:

La doble hermenéutica implica que las relaciones [entre las personas] son dialógicas... los "descubrimientos" pueden ser tomados por aquellos a cuya conducta se refieren, no es un fenómeno que puede o podría ser marginado, sino que es constitutivo de su naturaleza. Es el punto capital de conexiones entre dos mundos posibles en que las ciencias sociales relacionan sus resultados con la sociedad misma (Giddens, 1982, p.17).

Estos son nuestros fundamentos para permitirnos realizar una interpretación de las reflexiones de Lourdes, y es en ese camino que le propusimos donde se hacen evidentes las huellas que la marcaron para ser hoy quien es como persona y como profesional. $\mathrm{Al}$ respecto, Alsina y Batllori expresan:

La toma de conciencia de estos puntos fuertes y débiles es un paso fundamental en la construcción autorregulada del propio perfil docente, ya que ello les permite señalar diversos aspectos susceptibles de ser mejorados, es decir, sus expectativas (2015, p.8).

Para narrarse en este diario Lourdes debió muchas veces objetivarse, la mirada introspectiva que permite la respuesta, la reflexión. En esta línea de pensamiento los mismos autores señalan que es esencial para el docente reflexivo estructurar sus experiencias, compartirlas y que sean capaces de conocer y comparar sus narraciones con otros profesionales para poder descubrir nuevos caminos. Hablan de un aprendizaje como proceso social e interactivo tal como nos muestra Lourdes; personas con habilidades, expectativas y experiencias diferentes, con ritmos distintos, entran en contacto en ese momento mágico de la clase. Y es de esas interacciones que la docente va creando su propio perfil enriquecido por los otros y a la vez enriqueciendo a quienes recorren ese camino en las aulas.

En su diario Lourdes alude a la transformación que surge a partir de la reflexión, lo que nos lleva a lo que Connelly y Clandinin (1990) plantean respecto a la narrativa entendida tanto como método y como objeto de investigación. En esta línea, deberíamos denominar a nuestra investigación como "la historia de Lourdes" analizada desde la perspectiva narrativa. Los autores plantean que las personas "viven historias" y "cuentan historias" de sus vidas, mientras que los investigadores sociales describen esas vidas recogidas a través de las historias que les contaron y escriben narrativas de esas experiencias. La narrativa es un camino de caracterización de las experiencias humanas. En esta historia donde Lourdes se narra y nosotras la re-narramos para interpretarla, se da el fenómeno que los autores caracterizan en referencia al intercambio de relaciones que se generan a partir del desarrollo de estos textos narrativos. Ella narra, nosotras la interpretamos, empatizamos con sus vivencias, y al trabajar colaborativamente sobre esa narrativa, sentimos que también nuestras relaciones interpersonales como investigadoras se entrelazan. Se da una interpenetración de esferas de experiencia de distintas personas relacionadas con el proceso de investigación. Vemos, como dicen los autores, la importancia de que todos los involucrados tengan voz dentro de la relación, donde la distancia o separación no caracteriza el conocimiento conectado. Es el "juego de creer" que implica un proceso de autoinserción en la historia del otro como una forma de conocerlo y darle voz (Connely y Clandinin, 1990, p.4). 
Consideramos que hemos cumplido con los objetivos propuestos para este trabajo: la lectura y deconstrucción del diario de Lourdes, el proceso de interpretarla y re-narrarla ha dado cuenta de las transformaciones que ella misma manifiesta. La significatividad de su trabajo se pone de manifiesto en la búsqueda de estrategias no sólo innovadoras para la enseñanza del inglés sino también se expresa en sus esfuerzos por la integración y el reconocimiento del otro-alumno como persona, transformando su práctica profesional en una práctica significativa a nivel individual y relevante de manera social.

En palabras de Sigrun Gudmundsdottir "valores y narrativas están inextricablemente entrelazados... nos ayudan a interpretar el mundo... son instrumentos de interpretación que constituyen...una perspectiva selectiva desde la cual contemplamos al mundo que nos rodea (1998, p. 9). Hay en la historia de Lourdes algo de esto, vemos cómo su narrativa la ayuda a autodescubrirse y repensarse como hemos dicho varias veces, con toda la variedad y riqueza que esto conlleva, y llevarnos al corazón del saber pedagógico sobre contenidos que enriquecen las cuatro dimensiones de las narrativas según Shulman (1987) citado por Gudmundsdottir (1998), que son "la experiencia práctica, la interpretación, la reflexión y la transformación".

Así pensamos que podemos cerrar este camino que desandamos para entenderlo. Y creemos que salimos enriquecidas del mismo porque nos permitió vivenciar desde los ojos de Lourdes un relato que contribuye plenamente a la investigación educativa. Esta narración ha sido un proceso de mediación natural entre fenómenos de la vida cotidiana y de la vida en las aulas, donde cultura, individuos e instituciones nos mostraron un paisaje único (Gudmundsdottir, 1998).

Narrativas para comunicarnos, narrativas para entendernos, narrativas para compartir, narrativas para transformarnos...

\section{ReFERENCIAS}

Alsina, A. y Batllori R. (2015). Hacia una formación del profesorado basada en la integración entre la práctica y la teoría: una experiencia en el Practicum desde el modelo realista. Revista de Investigación en la Escuela, 85, 5-18.

Bartlett, F. (1932). Remembering: A Study in Experimental and Social Psychology. Cambridge: Cambridge University Press.

Bengtsson, J. (1995). What is Reflection? On reflection in the teaching profession and teacher education. Teachers and Teaching: Theory and Practice, 1(1),23-32. http://dx.doi.org/10.1080/1354060950010103

Bolívar, A., Domingo, J. y Fernández, M. (2001). La investigación biográfico-narrativa en educación. Madrid: La Muralla.

Bruner, J. (1991). Actos de significado. Más allá de la revolución cognitiva. Madrid: Editorial Alianza.

Calderhead, J. (1989). Reflective Teaching and Teacher Education. Teaching \& Teacher Education, 5(1), 43-51.

Connely J. \& Clandinnin, M. (1990). Stories of Experience and Narrative Inquiry. Educational Researcher, 19(5), 2-14.

Dewey, J. (1998). Democracia y Educación. Una Introducción a la Filosofía de la Educación. Madrid: Ediciones Morata.

Erickson, F. (1989). “Métodos cualitativos de investigación sobre la enseñanza”. En M. Wittrok (Ed.). La investigación de la enseñanza II. Métodos cualitativos de observación (pp. 203-247). Barcelona: Paidós

Fenstermacher, G. y Richardson, V. (2005). On Making Determinations of Quality in Teaching. Teachers College Record, 107, 186-213.

Giddens, A. (1982). Profiles and critiques in social theory. Hermenéutica y Teoria Social. University of California Press.

Gudmundsdottir, S. (1998). La naturaleza narrativa del saber pedagógico sobre los contenidos. En H. McEwan y K. Egan (Comps.). La narrativa en la enseñanza, el aprendizaje y la investigación. Buenos Aires: Amorrortu

Hyvärinen, M. (2010). Revisiting the Narrative Turns. Life Writing, 7(1), 69-82. Recuperado de http://www.uta.fi/ yky/yhteystiedot/henkilokunta/mattikhyvarinen/index/Revisiting\%20the\%20Narrative\%20turns.pdf

Jackson, P. (2002). Práctica de la enseñanza. Buenos Aires: Amorrortu Editores

Litwin, E. (1997). Las configuraciones didácticas. Una nueva agenda para la enseñanza superior. Buenos Aires: Paidós. 
Polkinghorne, D. (1988). Narrative Knowing and the Human Sciences. Albany: SUNY Press.

Porta, L y Sarasa, C. (2014). Resignificar la buena enseñanza desde la voz de docentes memorables en educación superior confrontada con Ortega y Gasset y otros académicos. Profesorado: Revista de currículum y formación del profesorado. Recuperado de http://www.ugr.es/local/recfpro/rev181COL6.pdf

Shulman, L. S. (1987). Knowledge and teaching. Foundations of a new reform. Harvard Educational Review, 57(1), $1-22$.

Souto, M. (2016). Pliegues de la formación. Sentidos y herramientas para la formación docente. Rosario: Homo Sapiens. van Dijk, T. (1997a). Estructuras y funciones del discurso. Madrid: Siglo XXI.

van Dijk, T. (1997b). Racismo y análisis crítico de los medios. Barcelona: Paidós.

Van Langenhove, L., \& Harré, R. (2016). Posicionamiento y autobiografía: el relato de vida. Revista de Educación, (9), 77-96. Recuperado de https://fh.mdp.edu.ar/revistas/index.php/r_educ/article/view/1900

\section{Anexo}

\section{REFLEXIÓN}

Marta Souto en su libro Pliegues de la Formación de 2016, inicia su relato autobiográfico de manera narrativa con un objetivo bien claro que plantea al inicio y que es pensarse como sujeto en formación y a la vez como formadora. Desde ahí es que busca en sus propias huellas aquello que la marcó en estos dos sentidos como sujeto. Ella dice textualmente,

[...] bucear en el pasado las motivaciones, las marcas, las huellas que otros trazaron y que se inscribieron en mí, dejando el interés profundo por la formación, su estudio y sus prácticas; romper el silencio de la página en blanco para, contando mi historia, invitar a los lectores a que escriban la suya. A que indaguen en ella... ( $\mathrm{p} 25)$

Teniendo en cuenta esto que plantea la autora es que les pedimos una escritura narrativa en forma de Diario Reflexivo.

Nuestro objetivo es ver de qué manera el producto de las entrevistas y el grupo focal que realizamos el año pasado, se han puesto de manifiesto en el trabajo cotidiano con un formato más explícito a partir haberlo puesto en palabras.

Este diario está dividido en dos momentos. Uno es introductorio y permite reflexionar acerca de las entrevistas en las que participaste para nuestra investigación y el otro es específico, directamente relacionado con tu trabajo en y para el aula. Te sugerimos seguir el orden de los mismos y pasar al segundo una vez finalizado el primero.

Primer momento

Escribe un relato en torno a cómo contribuyó lo narrado en la entrevista biográfica y en el grupo focal en tu propia práctica.

\section{Segundo momento}

Te pediremos ahora que elijas un grupo de alumnos en particular que, por algún motivo, consideres potente al momento de prepararles las clases o al estar frente a ellos. Durante dos semanas, al finalizar la clase del grupo seleccionado reflexiona y toma nota de aquellas cuestiones que puedan dispararse de las preguntas planteadas a continuación. Debés tener presente que las preguntas no forman un guión inalterable a responder, sólo actúan como orientadoras de aquellas cuestiones que buscamos que salgan a la luz en tu relato.

1. ¿Por qué elegiste este grupo en particular? ¿Cuáles son los elementos potentes que te condujeron a dicha elección? 
2. ¿Consideras que la reflexión en torno a tu biografía escolar a partir de las entrevistas en las que participaste para nuestra investigación ha modificado el diseño y puesta en marcha de la clase elegida o de tu propia práctica? ¿de qué manera?

3. ¿Qué similitudes o diferencias encontrás entre la clase seleccionada y tu biografía escolar?

4. En tu entrevista y en el grupo focal revelamos tu perfil de docente activo, contenedor y empático, ¿qué modificaste de tus prácticas luego de que este aspecto se hiciera evidente? ¿reforzaste tu trabajo en este sentido?

5. Sentite libre de usar este último espacio para narrar experiencias y sentimientos de tu práctica docente que desees compartir, que se hayan manifestado a partir de nuestros encuentros el año pasado.

¡Muchas gracias!

Instrumento diseñado para el Proyecto Huellas, GIEEC, CIMED, Facultad de Humanidades, UNMdP

\section{Notas}

1 Alumnos que resuelven las actividades rápidamente.

2 Cuentacuentos 